\title{
Barriers and Recommendations for Implementing the Baby-Friendly Hospital Initiative in Cairo University Hospitals: a Qualitative study
}

\author{
Marwa Rashad Salem, Ola A Mostafa, Hoda Ibrahim Ibrahim Rizk
}

Department of Public Health and Community Medicine, Faculty of Medicine, Cairo University, Egypt

\begin{abstract}
Background: University hospitals face many challenges on their way to achieving Baby friendly hospital imitative (BFHI) as they carry a dual role of teaching and service provision. Objective: to estimate health care providers' perspectives for implementing the BabyFriendly Hospital Initiative in Kasr Al-Aini- Obstetrics and Gynecology Hospital. Method: A qualitative exploratory study was performed in the Gynecology and Obstetrics Department, as well as the neonatal intensive care Unit (NICU) at Cairo University Hospital. A semistructured interview was used to get data from a purposive sample of 26 consented participants: 9 physicians, 15 nurses, and 2 medical staff with managerial positions. Results: Responses were analyzed using thematic content analysis. The priorities identified by thematic analysis were barriers and recommendations for Baby-friendly hospital initiative implementation from the perspective of health care providers. All interviewees cited lack of staff training and high turnover rate of health care providers, lack of resources, heavy workload in addition to lack of time spent with new mothers especially those with cesarean deliveries or with health problems of mothers or babies that lead to early mother-infant separation as the main barriers. All interviewees recommended on-going training and the use of audiovisual aids. Conclusion: Lack of staff training, heavy workload per staff, and lack of resources required for providing training courses and educational materials were the main barriers reported by the participants to proper BFHI implementation. Strong external support and incentive are required in order to enhance the implementation of BFHI.
\end{abstract}

Keywords: Baby-friendly hospital, barriers, recommendations, qualitative, breastfeeding

Corresponding author: Marwa Rashad Salem E-mail: mr80002000@yahoo.com

\section{Introduction}

World Health Organization/United Nations International Emergency Children's Fund (WHO/UNICEF) recognized the importance of the Baby-friendly Hospital Initiative (BFHI) to ensure sound infant and young child feeding. BFHI gives direction on the implementation, training, monitoring, assessment, and re-assessment of the Ten Steps to Successful Breastfeeding and the International Code of Marketing of Breast-milk Substitutes. ${ }^{1}$ The program provides certification to institutions meeting the 10 Steps, as evaluated by a board of outside observers. ${ }^{2}$ Several studies proved that BFHI principles implementation had a beneficial effect on breastfeeding rates and duration, child health, and quality of maternity services. ${ }^{3}$ Breast feeding promotion and continuation for two years not only lower the rising trends of overweight and noncommunicable diseases (NCDs) worldwide, but also help countries to achieve the sustainable development goals (SDGs). ${ }^{4,5}$

No. 2

April $\mathbf{2 0 2 0}$ 
Worldwide, all countries have been implementing $\mathrm{BFHI}$, with varying degrees of success. However, the BFHI has been facing many challenges with implementation and designation of hospitals as baby friendly over the past decade. A global report on national implementation by WHO 2017 showed that out of a total of 117 countries, $86 \%$ reported that they had implemented the BFHI and $71 \%$ reported that they had a BFHI program as of 2016-2017. However, only one in five had ever designated more than one-half of their maternity facility as Baby-Friendly, particularly in the Eastern Mediterranean Region (EMR). ${ }^{6}$ In the United States, of the 3281 facilities that give maternity care, 203 are certified as Baby-Friendly. Only $8.4 \%$ of newborns born in the United States are born in BabyFriendly facilities. $^{7}$

Despite the legislative acts and ministerial decrees for urging hospitals to become Baby-Friendly and abide by the Code ${ }^{[8]}$, Egypt is one of the countries among EMR that had low and declining BFHI implementation as reported by a study performed by Al-Jawaldeh and Abul-Fadl. ${ }^{9}$ According to EDHS 2014,breastfeeding practices in Egypt are not always optimal; 6 in 10 children were reported to have received a prelacteal feed after birth, only 4 in 10 children under 6 months of age were being exclusively breastfed, and around 3 in 10 children under 6 months were being bottle fed. The majority of children are not exclusively breastfed for the recommended 6 months. Overall, only 40 percent of all children under age 6 months are being exclusively breastfed, and at age 4-5 months only 13 percent of children are receiving only breast milk. ${ }^{10}$ Kasr Al-Aini- Obstetrics and Gynecology Hospital $(\mathrm{Ob} \& \mathrm{GH})$ provides a tertiary level of health care. Additionally, being a teaching hospital, $\mathrm{Ob} \& \mathrm{GH}$ provides a wide spectrum of health services which include outpatient, inpatient and emergency services. In addition, it is a rich source of education and research for physicians and nurses. Healthcare practitioners in the hospital play a critical role in supporting and encouraging breastfeeding. So the implementation of BFHI in Kasr Al-Aini- Obstetrics and Gynecology Hospital as a teaching hospital will have a great impact on the future health care providers, which will affect the health of many children and their mothers. However, university hospitals face many challenges on their way to achieve BFHI designation as they carry a dual role of teaching and service provision. ${ }^{11}$

The current study has for objective to estimate health care providers' perspectives for implementing the BabyFriendly Hospital Initiative in Kasr AlAini- Obstetrics and Gynecology Hospital.

\section{Method}

Study design and settings - A qualitative exploratory study was performed in the Cairo University Hospital (Kasr Al-Aini); a university-affiliated teaching hospital in Egypt. The Departments in the Ob\&GH contain 400 beds, receive 250 women daily on average, and assist around 36,500 deliveries per year. Mothers who give birth in the hospital are discharged within 24 hours if there are no complications. The study took place over 4 months' duration from September- October 2018.

Sample size and sampling technique - A purposive sample of 26 consented participants: 9 physicians, 15 nurses, and 2 medical staff with managerial positions in the Gynecology and obstetrics hospital and the neonatal intensive care unit (NICU) at Cairo University Hospitals. In depth interviews were used to get the required data from the study participants until data saturation. ${ }^{12}$ Health care providers were 
selected based on their years of work in the hospital and their ability to provide an informed perspective on BFHI implementation. Inclusion criteria: health care providers with a minimum of one-year of work experience in obstetrics \& gynecology department or NICU at Cairo University Hospital; a period where they were supposed to acquire the hospital policy, and those who approved to participate in the study.

In-depth semi-structured audiotaped faceto-face interviews of 45 minutes duration were performed. The interview questions were open ended. A pilot test was used, followed by minor modifications of the interview forms.

Participants were asked to provide their view about the barriers and recommendations for BFHI implementation. A systematic review of literatures $^{13,14,15}$, and meetings with key informants guided the development of the interview guide of the current study (Table 1).

\section{Statistical analysis}

The technique of thematic content analysis $^{12}$ was used for data processing. This analysis involves the following stages:

The first stage was pre-analysis to organize the materials in order to enhance understanding of the data. Therefore, there were thorough and repeated readings of data transcripts.

The second stage was the exploration of the material, which is systematic selection of the statements of the participants interviewed and the organization of categories.

The third stage: processing and interpretation of the results. Participants' quotes were used to illustrate the meanings in themes and summaries.

\section{Ethical Consideration}

Written informed consent was obtained directly from the participating health care providers before data collection and after an explanation of the study objectives and importance. All procedures for data collection were treated with confidentiality according to the Helsinki Declaration of biomedical ethics. ${ }^{16}$ To guarantee the anonymity of the respondents, they were identified by the letter I (interviewee) followed by a number according to the chronological order of the interviews (I01, I02, I03...I13). All administrative permissions were obtained from the hospital manager and from the head of the Obstetrics and Gynecology hospitals and NICU.

\section{Results}

All health care providers approached accepted to participate in the study ( $n=$ 26). The study group was composed of two medical staff who held management positions, nine physicians, and fifteen nurses working in the Gynecology and Obstetrics Department, and NICU at Cairo University Hospitals. Participants' age ranged from 28 to56 years. The majority was women and had 2-36 years of work experience on average. All nurses have attended breastfeeding courses. Two themes were revealed from the thematic analysis of the transcript: the first theme reflected the barriers to BFHI implementation, and the second one reflected the recommendations that participants thought they are needed for BFHI implementation as displayed in Table 2.

I-Barriers to BFHI implementation in the Gynecology and Obstetrics Hospital

All interviewees affirmed that there are different types of barriers that restrict proper BFHI implementation

Shortage of human resources and lack of motivation: All interviewed health care 
providers cited a shortage in staffing, especially nurses as a barrier. One nurse mentioned: "There have been no incentives to recognize our hard work". On the other hand, high turnover rate among appointed nurses was mentioned by the nurses. This was due to the low salary and the weak potentials for financial increments. All new nurses were hired on temporary contracts. All interviewees universally agreed upon "More nurses are needed".

Limited opportunities for training on BFHI:

Physicians expressed their views towards training programs in BFHI as incompetent "Training inadequately prepared us for work in resource-poor settings". One physician delineated his participation in training programs at the Faculty of Medicine Cairo University. He said that the first hospital training course, started in June 2009, included a series of 6 sessions taught over one-week period. Another health care provider received breastfeeding courses in 2004, included a series of two sessions taught over two days about BFHI. In addition, all respondents affirmed that they do not have access to regular refresher courses to reinforce their learning, provide practical demonstrations, and keep them up to date. They identified specific areas that are not satisfied with their nutrition education: nutrition-counseling, infant and young children feeding, breastfeeding, and how to implement the BFHI. The head nurse of the NICU mentioned that all nurses working in the NICU had participated in at least one training course in Breastfeeding. She added "nurses need ongoing continuous training in order to keep up the excellent abilities they have". "Nurses attended more training compared to physicians. There is, however, rapid turnover of nurses which means that such training is needed on a continuous basis" one nurse said.

Lack of time to apply the BFHI Ten steps: All interviewed health care providers agreed "It is the workload "Sometimes tasks are more difficult when you have to do everything very fast for too many patients in a very limited time". Time pressure also restricts the health workers' ability to provide skin to skin contact. Several participants felt that working according to BFHI standards needed more work time situations, such as dealing with complicated cases or cesarean deliveries. With respect to skin-to-skin contact after cesarean section, participants mentioned that nurses had to stay in the theatre beside mothers for a while. But she should return quickly to the labor room or maternity unit to execute other tasks. In addition, the early separation of mothers and infants posed another barrier to maternal learning, early skin to skin contact; and hence early initiation of breastfeeding. Also, neonatal problems that necessitate their admission to the NICU caused mother-infant separation. A nurse unit manager noted particularly: "It's not that easy, in times of staff shortages, no one wants to carry additional duties". Health care providers explained that due to limited time with patients, it is difficult to provide all the 10 steps. One physician said, “I think we're very limited in what we can do as physicians in the set-up of obstetric and NICU because... it's complicated and requires behavior intervention. There's really not enough time for that". Physicians focus on immediate medical concern instead of the more distant concern of teaching breastfeeding and how to manage related issues and concerns. As regards the establishment of breastfeeding support groups (step 10), “due to the insufficient number of staff in Kasralainy, outreach service to the 
community is not available and difficult

to be established" one nurse said.

Table (1) In-depth interview guidelines

\begin{tabular}{lll}
\hline \multicolumn{1}{c}{ Items } & \multicolumn{1}{c}{ Interview guidelines } \\
\hline Personal Information of the interviewees & - & $\begin{array}{l}\text { Role in the organization., experience years, sex, } \\
\text { education and training }\end{array}$ \\
\hline $\begin{array}{l}\text { Barriers and Recommendations for BFHI } \\
\text { implementation }\end{array}$ & What is your general opinion about the BFHI? \\
& $-\quad$ What are the barriers to BFHI implementation? \\
& What are your recommendations for BFHI \\
implementation in the hospital?
\end{tabular}

Table (2) Barriers and recommendations reported by health care providers to BFHI implementation in the Gynecology and Obstetrics Hospital.

\begin{tabular}{ll}
\hline \multicolumn{1}{c}{ Categories } & \multicolumn{1}{c}{ Subcategories } \\
\hline I. Barriers to BFHI & - Shortage of human resources and lack of motivation \\
implementation in the Gynecology & - Limited opportunities for training on BFHI \\
and Obstetrics Hospital & - Lack of time to apply the BFHI Ten steps \\
& - Shortage of health education materials \\
& - Mothers-centered barriers \\
\hline
\end{tabular}

II. Recommendations by health care - Skill building and Resources

providers to facilitate the implementation of - Updating knowledge and continuous education BFHI

Shortage of health education materials: Health care providers pointed out that there is shortage in visual teaching materials, resources for demonstrations and information booklets for nurses regarding the 10 steps. Specifically, there was shortage in breastfeeding charts, posters (especially showing local foods in food groups and demonstrating hygienic practices during preparing food for pregnant and lactating mothers as well as for infant weaning), information leaflets and booklets, picture information cards. On the other hand, three nurses said that "women are always interested in hearing them talk or watching health education videos and they are less interested in visual materials."

Mothers-centered barriers: Health care providers recognized that "BFHI implementation is a complex issue controlled by many internal and external factors." This concept made them understand that there are many barriers caregivers face in trying to comply with the 10 steps. It is important to acknowledge that people had prior knowledge and beliefs which set specific culturally grounded or traditional rooted beliefs. This included the belief that continued breastfeeding after getting pregnant could cause kwashiorkor in the breastfeeding child. "The potential conflict between health workers' counseling and cultural beliefs and perceptions is more general, and has been reported". One physician said.

II. Recommendations by health care providers to facilitate the implementation of BFHI

Skill building and Resources: Health care providers affirmed the need for "More skill-building in Breastfeeding". Practical training in BF is crucial for acquiring the skills of teaching breastfeeding to expectant and new mothers. Most interviewees believed that physicians and nurses have the greatest need of understanding of Breastfeeding. With regards to service delivery, there is a general consensus that BFHI needs to be strengthened by providing them with the

No. 2

April 2020 
required human resources and provision of educational materials to support BFHI related activities. All interviewees pointed out the importance of developing health education materials to support the application BFHI. One nurse said, 'Healthcare workers' involvement and consultation is essential fo rthe development of health education materials".

The breastfeeding Policy should be available in all areas serving mothers/infants. The policy should cover ten steps and include a summary of the Code. Educational materials for promoting Breastfeeding that cover the ten steps should be displayed in simple illustrations and should not be supported by companies producing breast milk substitutes. Staff should explain the negative effect of breast-milk substitutes (BMS) and the marketing of BMS to the Mothers during the ANC visits.

Prenatal education about breastfeeding should be displayed and available to all mothers attending antenatal care services. The written or displayed educational materials should be mother-friendly and should emphasize the benefits of breastfeeding and the negative effects of artificial feeding.

Updating knowledge and continuous education: Health care providers demanded infant feeding education. One physician said, "Nutrition information needs to be patient-friendly, including the applied aspects of breastfeeding and complementary feeding".

They recommended continuous training through nutrition seminars. Their training suggestions included the availability of nutritionist and a lactation consultant in the Gynecology and obstetrics clinics. One health care provider said, "Nurses need to know what they can tell caregivers to improve their infant feeding practice ... the specific concrete advice they can give them regarding their infant feeding where to feed when to feed, and how to feed."

Most health care providers suggested the following topics for education: exclusive breastfeeding, complementary feeding for 6-24-month-old children, correct technique of positioning and attachment to the mother, breast milk expression and, as well as counseling and communication skills.

\section{Discussion}

The current study was performed to explore barriers and recommendations for implementing the Baby-Friendly Hospital Initiative in Cairo University Hospitals from health care provider's perspective in the Obstetrics and Gynecology Department; as well as the neonatal intensive care Unit (NICU). The main barriers reported by the interviewed staff were lack of staff training and high turnover rate of health care providers, lack of resources, heavy workload in addition to lack of time spent with new mothers especially those with cesarean deliveries or with health problems of mothers or babies that lead to early mother-infant separation.

Staff training of all health care providers who come in contact with mothers and their babies is important to ensure their compliance and commitment for BFHI implementation. It was shown to increase exclusive breastfeeding rates both at discharge and afterwards. ${ }^{17}$ The current study revealed that one of the most important barriers to BFHI implementation was insufficient staff training compared to the recommendations. There is a real need for breastfeeding training courses especially to physicians; where only two of the interviewed physicians reported attending previous training courses. A higher percentage of the interviewed nurses reported attending a training course 
compared to physicians. Refresher courses are also required in order to retain the acquired skills and to deal with the problem of high turnover rates encountered in Kasralainy hospital among nurses; which poses another barrier regarding policy communication to all staff. Similar barriers were met in another study performed in New Zealand ${ }^{17}$ and many other countries all over the world as reported by WHO; where the capacity building of facility staff in maternity facilities was cited as a significant challenge. ${ }^{6}$ About half of the 200 trained health care providers in New Zealand study left the facilities and were replaced by a similar number of untrained staff. ${ }^{16}$ Building and strengthening a sustainable system should be targeted rather than counting on committed individuals. Continuity of BFHI interventions would be adversely affected when these individuals leave the facility. ${ }^{3}$

Kasr-Al-Ainy hospital is a large tertiary care hospital with large access (free of patient charges) to hospital and maternity services. The hospital receives a large number of patients from all governorates; therefore, the workload is heavy especially with the limited manpower, low salary, and limited incentives. This further lowered the motivation of health care providers to deliver the required skills on initiation of breastfeeding such as skin to skin contact immediately after delivery to the mothers. Similar barriers to a shortage of resources and lack of time were encountered by another study in Austria. ${ }^{15}$ Another encountered barrier reported by health care providers in the current study was the problem of high-risk deliveries, cesarean deliveries, sick mothers, and sick babies sent to the ICU; which created a necessity for the staff to acquire a skill to ensure safety for those mothers and babies first and then provide the necessary education for early breastfeeding initiation. This was a barrier to reach the target of $75 \%$ exclusive breastfeeding rates at discharge.

Early separation of mothers and infants posed another barrier to maternal learning, early skin to skin contact; and hence early initiation of breastfeeding. Separation in the current study was reported in cases of cesarean delivery, neonatal problems that necessitate admission to the NICU, or for security reasons. A review study performed in Canada involving studies in the USA, United Kingdom, and Australia viewed interference with maternal-infant contact as a commonly reported barrier. ${ }^{18}$

Lack of resources allocated to provide health education materials was another important barrier, coinciding with the finding of other studies performed in New Zealand hospitals ${ }^{17}$, USA, UK, and Australia. ${ }^{18}$

According to WHO, fostering the establishment of breastfeeding support groups (step 10) is one of the barriers met by maternity facilities in many countries [3]. The current study is not an exception. Due to the insufficient number of staff in Kasr-Al-Ainy, outreach service to the community is not available. In order to implement BFHI, considerable effort is needed to ensure that staff training is available and accessible to all staff. External motivations are required in the form of providing appropriate incentives and resources.

The strength of this study is the qualitative approach that provided valuable insights into health care provider's perspectives regarding the implementation of BFHI. The current study findings should be viewed with respect to the following limitation: It involved interviews with health care providers, thus reflected the barriers to implement BFHI from their perspective only. Further research is 
required to assess the barriers from the recipients' perspective by interviewing mothers during antenatal and early postnatal care.

\section{Conclusion}

This study viewed lack of staff training, heavy workload per staff, and lack of resources required for providing training courses and educational materials as barriers to proper BFHI implementation. Cairo university hospital is required to overcome these barriers. In order to facilitate the sustainable implementation of BFHI, strong external support and incentives are required. This support can be in the form of funding for training courses. Training of trainers among health care providers (TOT) could ensure training sustainability and thus facilitate BFHI implementation.

Conflict of interest: None declared

Acknowledgment: We thank the chairmen of the Obstetrics and Gynecology hospital and NICU for conducting this study. We also thank thehealth care providers for their participation in this study.

\section{References}

1. International Code of Marketing of Breastmilk Substitutes. Geneva: World Health Organization; 1981 (http://www.who.int/nutrition/publications/cod e_english.pdf).

2. Baby- Friendly USA. Guidelines and Evaluation Criteria for Facilities Seeking Baby-Friendly Designation. Sandwich, MA: Baby-Friendly, USA; 2010

3. WHO (2018) Implementation Guidance: Protecting, Promoting and Supporting Breastfeeding in Facilities Providing Maternity and Newborn Services-The Revised BabyFriendly Hospital Initiative. World Health Organization, Geneva.
4. Al Shehri, A.; Al Fattani, A.; Al Alwan, I, Obesity among Saudi children. Saudi J. Obes. 2013, 1, 3-9. [CrossRef]

5. Lim, S.S.; Allen, K.; Bhutta, Z.A.; Dandona, L.; Forouzanfar, M.H.; Fullman, N.; Gething, P.W.; Goldberg, E.M.;Hay, S.I.; Holmberg, M.; et al, Measuring the healthrelated sustainable development goals in 188 countries:A baseline analysis from the global burden of disease study 2015. Lancet, 2016, 388, 1813-1850. [CrossRef]

6. World Health Organization: National Implementation of the Baby-friendly Hospital Initiative, 2017. Geneva. Licence: CC BY-NCSA 3.0 IGO.

7. Baby Friendly USA. Find Facilities. 2014. Available at: http://www.babyfriendlyusa.org /find-facilities. Accessed October 10, 2014.

8. Ministry of Health and Population. Ministerial Decree 36/2014; Ministry of Health and Population:Cairo, Egypt, 2014.

9. Al-Jawaldeh A and Abul-Fadl A, Assessment of the Baby Friendly Hospital Initiative Implementation in the Eastern Mediterranean Region. Children, 2018 5, 41; doi:10.3390/5030041

10.El-Zanaty and Associates/Egypt, and ICF International. 2015. Egypt Health Issues Survey 2015. Cairo, Egypt: Ministry of Health and Population/Egypt and ICF International 11.Abul-Fadl, A., Farghally, N., Ez-ElArab, H.S., Shandidi, M.R., Mostafa O., AlAttar, G., Bakr, I., ElArabi, E., Fakher, O., Hussien, S., Ibrahim, H., Menazae, E., Rashad, M., Sabbour, S., Yousef, N. and Elkaluby, E, A Multicenter Survey for Monitoring the BabyFriendly Initiative in 6 University Hospitals in Egypt (2017-2018): A Comparative Analysis. Open Journal of Social Sciences, 2018. 6, 293306. https://doi.org/10.4236/jss.2018.612026

12.Ritchie J, Lewis J, Qualitative research practice: a guide for social science students and researchers. Los Angeles, London, New York: Sage Publications; 2009.Google Scholar.

13.Dür W, Applying system theory of organisational change to health promotion interventions in schools. In: Samdal O, Rowling L, editors. The Implementation of Health Promoting Schools. Exploring the 
theories of what, why and how. London: Routledge; 2013. p. 34-50.Google Scholar 14.Christina C Wieczorek , Hermann Schmied, Thomas E Dorner and Wolfgang DürInternational Breastfeeding Journal 201510:3

15.Wieczorekc, Hermann Schmied H, Dorner $\mathrm{T}$ and Dür $\mathrm{W}$, The bumpy road to implementing the Baby-Friendly Hospital Initiative in Austria: a qualitative study . International Breastfeeding Jounal , 2015 F0:3 16.Declaration of Helsinki on EthicalPrinciples for Medical Research Involving Human Subjects, as adopted by the 52nd WMA General Assembly, Edinburgh, October 2013.

17.Moore T, Gauld R and Williams S, Implementing Baby Friendly Hospital Initiative policy: the case of New Zealand public hospitals. International Breastfeeding Journal 2007, 2:8

18.SemenicS,Childerhose $\mathrm{J}$, Lauzière $\mathrm{J}$, and Groleau D, Barriers, Facilitators, and Recommendations Related to Implementing the Baby-Friendly Initiative (BFI): An Integrative. Journal of Human Lactation ,2012 